\section{ENVIRONMENTAL MICROBIOLOGY}

\section{Pay dirt}

Cultured microorganisms have been the source of almost all characterized antibiotic-resistance genes. Yet these microorganisms represent only a tiny fraction of microbial diversity, limiting access to the potentially vast reservoir of undiscovered antibiotic-resistance determinants harboured by uncultured bacteria. A report published in Environmental Microbiology describes the use of metagenomic analysis to explore this reservoir in uncultured soil bacteria — an analysis that has revealed the presence of antibiotic-resistance genes considerably different from previously reported sequences.

The approach adopted by Jo Handelsman and colleagues was to isolate DNA directly from soil samples, clone the DNA and select for expressed antibiotic resistance in Escherichia coli. Four libraries were constructed containing 4.1 gigabases of cloned soil DNA and, from analysis of these libraries and two previously reported libraries, ten clones expressing antibiotic resistance were identified. From preliminary screening of these clones and subsequent sequence analysis, the authors identified nine aminoglycoside-resistance genes and one gene encoding a tetracyclineefflux protein. The sequence analysis also revealed that the resistance mechanisms used by these proteins included the inactivation of aminoglycoside antibiotics by phosphorylation and acetylation, as well as the efflux of tetracycline. Interestingly, nine out of the ten resistance proteins identified were found to have less than $60 \%$ identity to previously published sequences, clearly suggesting that soil microorganisms harbour antibiotic-resistance genes with significantly more genetic diversity than indicated by previous studies.

The study of Riesenfeld et al. provides an insightful snapshot of the power of metagenomic analysis in unlocking the biological potential of the uncultivatable bacterial community. Harnessing that potential in the search for new antibiotic entities remains the ongoing goal.

David O'Connell

\section{VIRAL EVOLUTION}

\section{Ordering fitness}

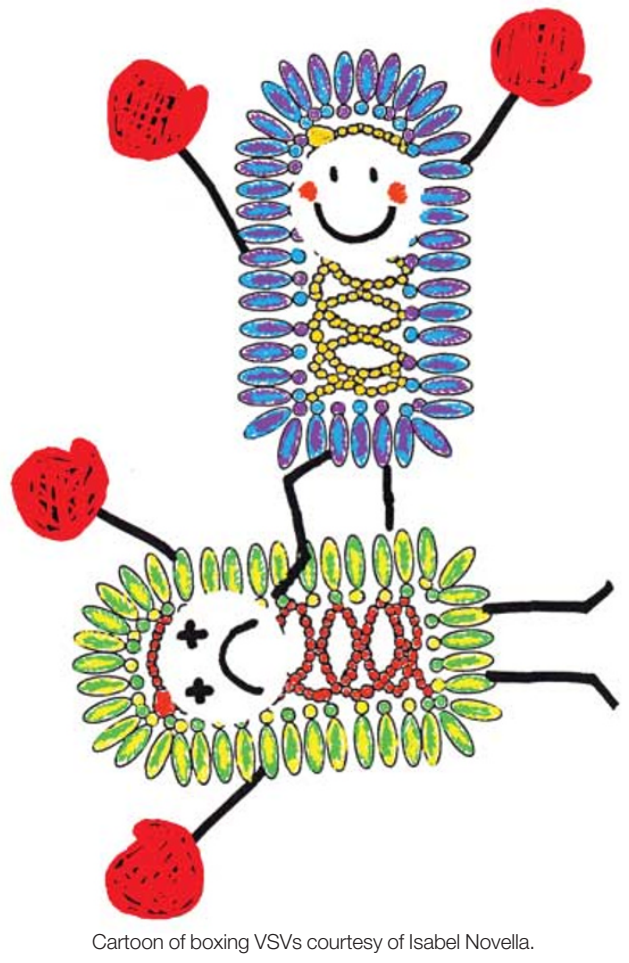

The order of genes in the vesicular stomatitis virus (VSV) genome is crucial for viral fitness, according to a new study published in the Journal of Virology.

Many concepts in evolutionary and population genetics apply to RNA viruses, and VSV, the prototype of the Rhabdoviridae, is a model system for evolutionary studies. Gene expression is controlled at the level of transcription in VSV and is determined by gene order - the genes that are nearest to the single $3^{\prime}$ promoter are the most highly transcribed.

Considering this mechanism of gene regulation, it is not surprising that gene order $\left(3^{\prime}-\mathrm{N}, \mathrm{P}, \mathrm{M}, \mathrm{G}, \mathrm{L}-5^{\prime}\right)$ is well conserved in VSV. However, previous experiments that rearranged the order of the VSV P, M and G genes yielded viruses that seemed to grow as well, or better, in vitro than wild-type (WT) virus.

Novella et al. initially confirmed that both the growth kinetics and the amounts of virus produced by infected cells were comparable between WT and rearranged VSV viruses. Assessing the fitness of the rearranged viruses was not as straightforward. A monoclonal antibody-resistant mutant (MARM) of VSV was selected (RU) that was as fit, in competition assays, as the WT virus. Competition assays were followed by selection and enumeration of this marked surrogate WT virus.
Competition studies showed that the fitness of each rearranged virus was reduced compared with RU. Intriguingly, fitness of the rearranged viruses improved with increasing multiplicity of infection (MOI, the average number of virus particles that infect a single cell).

At high MOIs the likelihood of co-infection of a host cell with both WT and a rearranged virus is increased. The authors favour the possibility that the dependence of increased fitness of rearranged viruses on the MOI is due to complementation of less-fit rearranged viruses by wild-type isolates. The fate of rearranged and other mutant viruses during infection might rely on complementation in co-infection, which is thought to be common in virus spread though infected hosts.

Closer inspection of rearranged VSV viruses shows that the wild-type virus is always the most fit, so it seems that evolution has selected the fittest genome after all.

Susan Jones

\section{(2) References and links}

ORIGINAL RESEARCH PAPER Novella, I. S. et al.

Fitness analyses of vesicular stomatitis strains with rearranged

genomes reveal replicative disadvantages.

J. Virol. 78, 9837-9841 (2004)

WEB SITE

Isabel Novella's laboratory:

http://www.mco.edu/micro/faculty/novella.html 